\title{
Model-based Resource Prediction for Multi-hop Wireless Networks
}

\author{
Yuan Sun $^{\dagger} \quad$ Xia Gao \\ $\dagger$ Department of Computer Science \\ University of California, Santa Barbara \\ \{suny, ebelding\}@cs.ucsb.edu
}

\author{
Elizabeth M. Belding-Royer ${ }^{\dagger} \quad$ James Kempf $^{\ddagger}$

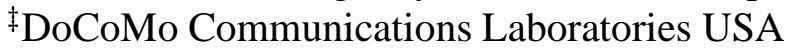 \\ \{gao, kempf\}@docomolabs-usa.com
}

\begin{abstract}
Ad hoc networks have been proposed for a variety of applications where support for real time, multimedia services may be necessary. This requires that the network is able to offer quality of service (QoS) appropriate for the latency and throughput bounds needed to meet the real time constraint. An important component for QoS provisioning is resource estimation and quality prediction. This paper describes a model-based resource prediction (MBRP) mechanism to support real time communication in multi-hop wireless networks. Specifically, we develop an analytical model for differentiated MAC scheduling protocols. The model can predict per-flow and system-wide throughput and delivery latency, thereby enabling admission control of the flows and providing an efficient network management utility. After describing the basic model, we propose Enhanced MBRP (EMBRP) for realistic network environments. Our proposed quality prediction method is beneficial in the deployment of a real ad hoc network where knowledge of resource allocation and consumption is needed to meet the service requirements. Analytical and simulation results show that EMBRP provides accurate flow quality prediction. The results also demonstrate the effectiveness of EMBRP as an admission control solution in multi-hop ad hoc networks.
\end{abstract}

\section{Introduction}

Wireless networking and multimedia content are two rapidly emerging technological trends. Among types of wireless networks, multi-hop ad hoc networks provide a fexible means of communication when there is little or no infrastructure, or the existing infrastructure is inconvenient or expensive to use. They are also useful in conjunction with infrastructured wireless networks to extend the coverage area of access points. With the development of ad hoc networks, we can anticipate that multimedia applications will be popular in scenarios where these networks are used.

One challenge of providing multimedia services in wireless networks is that certain quality of service (QoS) metrics should be satisfi ed. There has been signifi cant research on QoS provisioning in wired networks. For instance, Intserv [21] and Diffserv [10, 17] are two well-known approaches. In ad hoc networks, however, several unique characteristics make QoS provisioning more challenging. These characteristics include the shared wireless medium, mobility, and the distributed multihop communication.

Most QoS solutions for wired networks rely on the availability of precise resource utilization information for wired links. However, in ad hoc networks, all traffi c within a mobile node's transmission range contends for media access; the shared na- ture of wireless communication channels hence makes resource estimation more challenging. Multi-hop interference introduces further challenges to the problem, making it diffi cult to accurately determine the available resources. Without suffi ciently accurate estimation of channel utilization and prediction of fbw quality, i.e., throughput or transmission delay, it is diffi cult to provide multimedia services with satisfactory quality.

Service quality prediction is therefore an important building block for providing QoS in multi-hop wireless networks. It also enables effective admission control. The latter is important for ad hoc networks because these networks generally have limited resources, in terms of both device capabilities and available network bandwidth. If a fbw has rigid QoS requirements, a prediction of the achievable quality will prevent the waste of resources at both the source node and in the whole network if the network cannot support the fbw.

In this paper, we propose a model-based resource prediction (MBRP) scheme to provide fbw quality prediction for ad hoc networks. Our targeted network environment is multi-hop wireless networks where support of multimedia services is desired. To help meet the real time constraints, priority scheduling mechanisms at the MAC layer can be utilized in this environment [1, 13, 22]. For instance, a Voice over IP (VoIP) traffi c session has stringent real time constraints and therefore could be labeled high priority while other delay-tolerant traffi c can be given lower priority. Under this context, our model supports various differentiated MAC schemes with multiple priorities and provides estimation of both per-fbw and aggregated network-wide throughput and delay analysis. We further apply the basic MBRP analysis to a realistic network environment, i.e., unsaturated nodes and hidden terminal interference exist, and propose Enhanced MBRP (EMBRP) to improve the estimation accuracy.

Model-based prediction has two important benefi ts. First, it improves channel effi ciency by avoiding the waste of the network resources due to unprovisioned traffi c. By using the MBRP analysis, a fbw can check whether the network can support the real time requirements before it starts. Consequently, serviced fbws will meet the desired quality, thereby improving the channel effi ciency. Secondly, MBRP enables fexible admission control with a wide range of quality policies. This is especially important when service differentiation is supported in the network. The network quality policy can then be, for instance, to maximize the network-wide through- 
put, or to admit the maximum number of high quality fbws that can be supported. However, no matter what policy is required, the admission of a new fbw will affect ongoing traffi $\mathrm{c}$ because of the shared wireless channel. Because our model predicts the impact of the new fbw on both new and existing traffi c, flexible admission control can be achieved.

The remainder of this paper is organized as follows. Section 2 describes related work. Section 3 presents our proposed basic MBRP analysis and its improvement, EMBRP, for a realistic network environment. We then describe how an estimation module using MBRP can be integrated with existing routing schemes for multi-hop wireless networks in section 4 . The performance of our proposed approach is evaluated in section 5 , and fi nally section 6 concludes the paper.

\section{Related Work}

Resource estimation has been studied extensively in wired networks $[7,18,20]$. The bandwidth or latency of a path can be estimated through end-to-end probing techniques. For instance, the packet bunch technique $[7,18]$ measures the available bandwidth between a node pair by dividing the receiverACKed probing packets with the time interval between the fi rst and last received packets. Latency measurements between two nodes can be achieved through ping messages or any designated packets [20]. To deal with high network variability, multiple measurements are needed to achieve a better estimation.

In ad hoc networks, resource estimation in recently proposed QoS-aware routing protocols often takes advantage of statistical information provided by MAC layer. These solutions can be categorized into the following groups: active measurement, passive measurement, emulation-based and modelbased approaches.

Active measurement methods from wireless networks inherit the basic techniques for wired networks; however, the primary difference is that they are typically conducted in a hopby-hop fashion, due to the lack of information about the full path. Hence, they are often combined with the route acquisition process. For instance, SWAN [2] uses a request/response probe during route discovery to estimate bandwidth availability along a path. A ticket-based probing technique to measure link delay is proposed in [9]. Each probe accumulates the delay of the path it has traversed. One drawback of active measurements is that they are susceptible to network variability. Extra effort is required to dampen short-time variations and maintain measurement stability.

Passive measurement techniques leverage the unique characteristics of wireless networks through a collection of channel statistics at the MAC layer. For instance, Quiet Time Fraction is often suggested to predict the available bandwidth of a wireless channel by listening to the channel and measuring the fraction of time during which the channel is not in use [8, 24]. Packet forwarding latency is often measured by timestamps on RTS/CTS or DATA/ACK packets $[2,16]$. Compared to active measurement, passive methods have the advantage of less control overhead. However, they often do not fully consider the contentious nature of 802.11-based MAC access.
An emulation-based delay estimation method, Virtual MAC (VMAC) [3, 23], captures most of the aspects of a real MAC and operates in parallel to the real MAC protocol. However, no real packets are actually transmitted. Instead, the VMAC algorithm estimates the probability of collision if the real packet were transmitted. VMAC emulates real MAC behavior without introducing any communication overhead. However, one drawback of VMAC is that when multiple nodes simultaneously utilize VMAC estimation, they cannot detect the collisions that will occur because the packets are not actually transmitted.

Several analytical models for IEEE 802.11 have been proposed [2, 5, 15]. Bianchi [5] uses a discrete Markov chain model to capture the behavior of CSMA/CA channel multiplexing in IEEE 802.11 and derives the saturated throughput based on a constant and independent channel collision ratio $p$. However, it does not specify how to calculate $p$ in a given network topology. It also does not support priority-based differentiation schemes. A delay model for IEEE 802.11 is derived in [2] by assuming that the channel contention of competing fbws are Poisson distributed. The model also takes into account the effect of different $C W_{\min }$ and $C W_{\max }$ values. However, similar to [5], it does not specify detailed algorithms to estimate channel utilization and contention window size of each fbw, which is the key parameter for delay estimation. The model also does not support other differentiation schemes, such as different backoff ratio or backoff policies. A more detailed model to estimate channel utilization (based on which our model is constructed) and network throughput is provided in [15]. The advantage of this model is that it includes greater detail of IEEE 802.11. One limitation of the model is that it does not provide fbw-based estimation. It also does not support priority-based MAC schemes.

To summarize, these models do not fully consider different types of differentiation schemes used in IEEE 802.11. Because of their limitations, the schemes cannot provide a fbwlevel QoS estimation at the level of accuracy that is needed by admission control. These models also do not address issues such as random channel corruption, non-saturated nodes, or hidden terminals within carrier-sensing range. In contrast, MBRP provides per-fbw quality analysis as well as consideration of the above issues.

\section{Model-based Resource Prediction (MBRP)}

In this section, we propose a model-based resource prediction mechanism for multi-hop ad hoc networks. The primary objective of our mechanism is to provide accurate resource prediction for both new and existing traffi c. We fi rst describe the basic MBRP model in section 3.1. Then in section 3.2, we explain how MBRP can be applied to a realistic network environment and propose Enhanced MBRP (EMBRP).

\subsection{Basic MBRP Mechanism}

The basic premise of MBRP is to provide quality prediction for both ongoing traffi $\mathrm{c}$ and new fbws so that a correct fbw ad- 
mission decision can be made according to the quality of service policy of the network. Our model is based on the model of basic IEEE 802.11 DCF described in [2, 5, 15]. However, we extend existing work and make the following contributions:

- Development of a differentiated MAC scheme model with multiple priorities.

- Development of models for different priority-based backoff schemes.

- Estimation of both per-fbw and aggregated system-wide throughput and delay.

Hence, our model is more generic and has wider applicability than the previously proposed models. It can be used by admission control schemes for both delay and bandwidth sensitive applications. The admission decision can be modifi ed to either admit the maximum number of supportable high-priority fbws, or to achieve the maximum network throughput.

\subsubsection{Priority Scheduling Model}

To provide throughput and delay estimation, we fi rst need to model the node backoff behavior and analyze the channel utilization. Similar to previous work $[2,15]$, we assume that the time interval between two adjacent transmission attempts is exponentially distributed. As a result, the channel attempt rate is assumed to follow a Poisson distribution with an average rate of $\lambda_{c}$. We also assume that the channel collision rate, $p$, is constant and only relates to the current competing traffi c load $[2,5,15]$.

Let $A=\left\{a_{1}, a_{2}, \ldots, a_{s}\right\}$ be a set of fbws with different priorities, where $s$ denotes the total number of priority classes supported by the system, and $\forall a_{i} \in A, a_{i}$ is the number of fbws of priority class $i$. To maintain the clarity of the derivation, we let fbws with the same priority level have the same average packet length $F_{i}{ }^{1}$ and the same average backoff window size $L\left(a_{i}\right)$.

The current average channel attempt rate, $\lambda_{c}$, which indicates the number of transmission attempts in a time slot, can then be represented by

$$
\lambda_{c}=\sum_{i=1}^{s} \sum_{j=1}^{a_{i}} \frac{1}{b_{i, j}}=\sum_{i=1}^{s} \frac{a_{i}}{\bar{b}_{i}}=\sum_{i=1}^{s} \frac{a_{i}}{L\left(a_{i}\right)}
$$

where $b_{i, j}$ denotes the backoff window size of fbw $j$ with priority class $i$ and $\bar{b}_{i}=E_{j}\left[b_{i, j}\right]=L\left(a_{i}\right)$.

In contrast to previous work $[5,15]$, our intention is to support multiple priority levels. To this end, the channel attempt rate, collision rate, and backoff window size of different priorities must to be differentiated.

The channel attempt rate $\lambda_{c}$ in Eq. (1) includes the effect of all the fbws in the system. For each individual fbw with priority $i$, the attempt rate of the competing fbws is

$$
\lambda_{i}=\lambda_{c}-1 / L\left(a_{i}\right)
$$

The competing fbws include all other fbws except the given fbw itself. The transmission of the fbw is successful only

\footnotetext{
${ }^{1}$ Our derivation also hold for scenarios where fbws of the same priority have different packet sizes. Instead of forming equations for each priority, we need to form equations for each fbw. However, the basic principles are the same and the equations are still solvable.
}

when all competing fbws do not transmit. Hence, the collision probability of a fbw with priority $i, p_{i}$, is

$$
p_{i}=1-e^{-\lambda_{i}}
$$

Before calculating the average backoff window size, we need to fi rst decide what type of backoff scheme to use. The generic form of a priority-based backoff scheme is $C W_{n e x t}=$ $f_{a_{i}}\left(p_{i}, C W_{c u r r}, i\right)$. In the basic exponential backoff scheme of DCF, $C W_{\text {next }}=2 * C W_{\text {curr }}$. In our previous work [22], we proposed a series of priority-based backoff schemes that differentiate traffi $c$ with various priorities. One scheme is shown below as an example. A more detailed description of the schemes and a performance comparison can be found in [22].

Let $m$ be the maximum number of retransmissions. For the exponential backoff scheme, the probability that the $j t h$ collision occurs is

$$
c_{j}= \begin{cases}c_{0} \cdot p_{i}^{j} & 1 \leq j \leq m-1 \\ c_{0} \cdot \sum_{k=m}^{\infty} p_{i}^{k}=\frac{c_{0} p_{i}^{m}}{1-p_{i}} & j=m\end{cases}
$$

where $\sum_{k=0}^{m} c_{k}=1$, and we also have

$$
c_{0} \cdot\left(1+p_{i}+p_{i}^{2}+\cdots+\frac{p_{i}^{m}}{1-p_{i}}\right)=1 \Longrightarrow c_{0}=1-p_{i}
$$

Then for any priority class $i$, the average backoff window size during collisions is

$$
\begin{aligned}
& L\left(a_{i} \mid \text { backoff }\right)=b_{0} c_{0}+b_{1} c_{1}+\cdots+b_{m} c_{m} \\
& =\sum_{j=0}^{m-1} \frac{C W_{j}-1}{2} p_{i}^{j}\left(1-p_{i}\right)+\frac{C W_{m}-1}{2} p_{i}^{m}
\end{aligned}
$$

Now consider the following priority-based backoff scheme $C W_{j}=\left[2^{j}+p_{i} \alpha_{i}\right] C W_{\min }(\forall j \in[0, m])$ where $p_{i}$ is the collision rate and $\alpha_{i}$ is a constant associated with the fbw's priority class $i$ to differentiate the backoff ratio of different priority fbws. We have

$$
\begin{aligned}
& L\left(a_{i} \mid \text { backoff }\right)=\sum_{j=0}^{m-1} \frac{\left(2^{j}+p_{i} \alpha_{i}\right) C W_{\min }-1}{2} p_{i}^{j}\left(1-p_{i}\right) \\
& +\frac{\left(2^{m}+p_{i} \alpha_{i}\right) C W_{\min }-1}{2} p_{i}^{m} \\
& =\sum_{j=0}^{m-1} \frac{2^{j} C W_{\min }-1}{2} p_{i}^{j}\left(1-p_{i}\right)+\frac{2^{m} C W_{\min }-1}{2} p_{i}^{m} \\
& +\left[\sum_{j=0}^{m-1} \frac{\alpha_{i}}{2} p_{i}^{j+1}\left(1-p_{i}\right)+\frac{\alpha_{i}}{2} p_{i}^{m}\right] \cdot C W_{\min } \\
& =\frac{C W_{\min }}{2\left(1-2 p_{i}\right)}\left[1-p_{i}-p_{i}\left(2 p_{i}\right)^{m}\right]+\frac{\alpha_{i}}{2} p_{i} \cdot C W_{\min }-\frac{1}{2}
\end{aligned}
$$

where $C W_{\min }$ is the minimum contention window size. By adjusting $\alpha_{i}$, we can adjust the sensitivity of the difference of different priority classes with respect to the collision rate $p_{i}$.

Continuing the average backoff window size, $L\left(a_{i}\right)$, calculation, Eq. (7) gives the average backoff window size under the condition that the channel is sensed busy. IEEE 802.11 specifi es that the node transmits immediately without backoff if the channel is sensed idle for the DIFS period. Let $P_{f r e e, i}$ denote the probability of a free channel when the node attempts a 
transmission and all competing fbws are in the backoff stage, and let $P_{\text {busy }, i}$ denote the probability of a busy channel when at least one competing fbw is transmitting. Let $\bar{F}$ be the average packet transmission time:

$$
\bar{F}=\frac{\sum_{i=1}^{s} \sum_{j=1}^{a_{i}} F_{i j}}{\sum_{i=1}^{s} a_{i}}=\frac{\sum_{i=1}^{s} a_{i} \cdot F_{i}}{\sum_{i=1}^{s} a_{i}}
$$

Using the same assumption that the transmission attempt of competing fbws is of Poisson distribution with rate $\lambda_{i}$, and the inter-arrival time between two adjacent transmission attempts is of exponential distribution with average of $1 / \lambda_{i}$, we have

$$
\begin{gathered}
P_{\text {free }, i}=\frac{1 / \lambda_{i}}{\bar{F}+1 / \lambda_{i}} \\
P_{\text {busy }, i}=\frac{\bar{F}}{\bar{F}+1 / \lambda_{i}} .
\end{gathered}
$$

Then the average backoff window size $L\left(a_{i}\right)$ is

$$
\begin{aligned}
L\left(a_{i}\right) & =P_{\text {free }, i}\left(1-e^{-\lambda_{i}}\right) L\left(a_{i} \mid \text { backoff }\right) \\
& +P_{\text {busy }, i} L\left(a_{i} \mid \text { backoff }\right)
\end{aligned}
$$

Hence, we have derived the expression of the current channel attempt rate $\lambda_{c}$ as a function of average backoff window size $L\left(a_{i}\right)$ in Eq. (1), contending traffi c rate $\lambda_{i}$ for a node with priority $i$ as a function of $\lambda_{c}$ and $L\left(a_{i}\right)$ in Eq. (2), the expression of collision possibility $p_{i}$ as a function of $\lambda_{i}$ in Eq. (3), and fi nally, the expression of the average backoff window size $L\left(a_{i}\right)$ as a function of the collision possibility $p_{i}$ in Eq. (10).

For any given fbw set $a_{1}, a_{2}, \ldots, a_{s}$, we can obtain a derived $\lambda_{i}^{\star}, p_{i}^{\star}$ and $L\left(a_{i}\right)^{\star}$ by using a similar iteration algorithm as in [15]. The difference is that we need to calculate the variables for each priority separately. Specifi cally, as proved in [12], the expected number of collisions in a binary backoff algorithm grows asymptotically with $O(\log M)$, where $M$ is the number of active stations in the network. Hence, the initial backoff window size $L\left(a_{i}\right)^{(0)}$, in our scheme, is bounded by the following:

$$
L\left(a_{i}\right)^{(0)}<C W_{m i n} \cdot 2^{K \cdot \log \left(\sum_{i=1}^{s} a_{i}\right)}
$$

where $K(>0)$ is some arbitrary constant. Given $L\left(a_{i}\right)^{(0)}$ of each priority, which represents the largest backoff window size, we can fi rst obtain the channel attempt rate $\lambda_{c}$ by using Eq.(1 and based on that, calculate $\lambda_{i}^{(0)}$ and $p_{i}^{(0)}$ using Eq. (2) and (3), respectively. Then, by applying Eq. (10), we can calculate $L\left(a_{i}\right)^{(1)}$. The iteration repeats until the difference of two consecutive iteration values satisfi es $\left|L\left(a_{i}\right)^{(j+1)}-L\left(a_{i}\right)^{(j)}\right|<\epsilon$, where $\epsilon$ denotes some predefi ned small value. The iterative algorithm always converges as proved in Theorem 1 in [15].

\subsubsection{Throughput Model}

Given the current traffi $\mathrm{c}$ rate $\lambda_{c}$, the competing traffi c rate for each priority $\lambda_{i}$, the collision possibility $p_{i}$, and the average backoff window size $L\left(a_{i}\right)$, we now derive the throughput calculation for each priority fbw.

Let $B$ denote the capacity of the wireless link (e.g., 2Mbps). Because the packet transmission attempt is of Poisson distribution with rate $\lambda_{c}$, the inter-arrival time (the portion of wasted bandwidth) between two adjacent transmission attempts is of exponential distribution with average $1 / \lambda_{c}$. Considering that the average packet length (the portion of utilized bandwidth) is $\bar{F}$, the total utilized bandwidth, $B_{u}$, expressed as the ratio of link capacity $B$ is:

$$
B_{u}=B \times \frac{\bar{F}}{\bar{F}+1 / \lambda_{c}}
$$

Let $B_{i}$ be the bandwidth utilized by a fbw of priority $i$ and $\bar{B}_{u}$ be the sum of channel usage of all fbws. Then,

$$
\overline{B_{u}}=\sum_{i=1}^{s} a_{i} \cdot B_{i}
$$

Due to transmission collisions among competing fbws, $\overline{B_{u}} \geq$ $B_{u}$. Note that the portion of collided bandwidth is calculated multiple times in $\bar{B}_{u}$. More precisely, if there are $i$ fbws transmitting at the same time, this portion of the bandwidth is added $i$ times in the $\bar{B}_{u}$. Because the probability of $i$ simultaneous transmissions is $\left(\lambda_{c}^{i} / i !\right) e^{-\lambda_{c}}$, under the condition that there is a transmission,

$$
\begin{aligned}
& \bar{B}_{u}= \frac{\lambda_{c} e^{-\lambda_{c}}}{\left(1-e^{-\lambda_{c}}\right)} \cdot B_{u}+\frac{2 \times\left(\lambda_{c}^{2} / 2 !\right) e^{-\lambda_{c}}}{\left(1-e^{-\lambda_{c}}\right)} \cdot B_{u}+\ldots \\
&=\sum_{j=1}^{\infty} j \cdot\left(\frac{\lambda_{c}^{j}}{j !}\right) \cdot \frac{e^{-\lambda_{c}}}{1-e^{-\lambda_{c}}} \cdot B_{u}
\end{aligned}
$$

Notice that the fi rst item in Eq. (14) is the no-collision portion of $B_{u}$, which is also the overall throughput, $T$, of the wireless link. $T$ can be given by

$$
T=\frac{\lambda_{c} e^{-\lambda_{c}}}{\left(1-e^{-\lambda_{c}}\right)} \cdot B_{u}
$$

Now let's consider per-fbw throughput. For each priority fbw $i$, the channel utilization is proportional to its average packet length $\bar{F}_{i}$ and inversely proportional to its average waiting time between two adjacent transmissions. The average waiting time is the sum of its own backoff window size $L\left(a_{i}\right)$ and the total transmission time of other competing nodes during $L\left(a_{i}\right)$. Hence, the ratio of channel utilization between two different priorities $i$ and $j$ can be presented as:

$$
\frac{B_{i}}{B_{j}}=\frac{\left(L\left(a_{j}\right) \lambda_{j} \bar{F}+L\left(a_{j}\right)\right) \cdot F_{i}}{\left(L\left(a_{i}\right) \lambda_{i} \bar{F}+L\left(a_{i}\right)\right) \cdot F_{j}}
$$

For a total of $s$ priority fbws, $(s-1)$ independent equations of type of Eq.(16) can be listed. Together with Eq.(13), this set of equations can be used to calculate $B_{i}$ of each priority.

Finally, considering the collision loss of each priority, the effective throughput of each priority traffi $\mathrm{c}$ is then

$$
\bar{B}_{i}=\left(1-p_{i}\right) \cdot B_{i}=e^{-\lambda_{i}} \cdot B_{i}
$$

Figure 1 shows a comparison between the analytical and simulation results for the throughput (both per-fbw and aggregated) versus the number of fbws in a single broadcast region. The lines are the numerical results calculated using Eq.(15) and (17), whereas the symbols are the values obtained from simulation. We can see that the simulation results closely match the analysis, thereby verifying our model. 


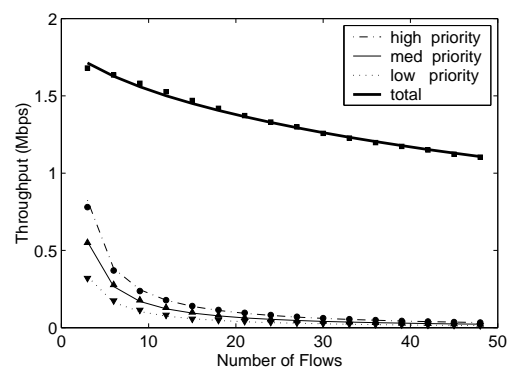

Figure 1: Throughput Model Verifi cation. $C W_{\min }$ is set to $32, m$ is set to 5 , and the packet size is set to 1000 bytes. The $\alpha$ values for different priorities are set to 1,8 and 16 .

\subsubsection{Delay Model}

We now derive the delay model based on the competing fbw traffi c rate $\lambda_{i}$ and the collision possibility $p_{i}$, as calculated in section 3.1.1.

Following the same analysis as in [23], let $d_{j}\left(a_{i}\right)$ denote the total deferred time during the $j$ th backoff for priority $i$. Because the backoff timer only decreases when the channel is idle, we have

$$
d_{j}\left(a_{i}\right)= \begin{cases}\bar{F}^{\prime}+k_{j} \bar{F}+b_{j} & j=1, \\ k_{j} \bar{F}+b_{j}+\bar{F} & j>1\end{cases}
$$

where $b_{j}$ is the backoff time of the $j$ th collision. $k_{j}$ is a Poisson random variable with average $\lambda_{i} \cdot b_{j}$ and denotes the number of packets that are sent during the $j$ th collision. $\bar{F}$ is the average packet length of the traffic and $\bar{F}^{\prime}=F / 2$ is the residual packet length that caused the collision on the fi rst try.

Hence, given the current attempt rate $\lambda_{i}$ and the collision possibility $p_{i}$ calculated using Eq. (2) and (3), the average value of the total accumulated deferred time for priority $i$, denoted as $d_{l}$, can be estimated as

$$
\begin{aligned}
d_{l} & =E\left[\sum_{j} d_{j}\right] \\
= & \sum_{l=0}^{\infty} E \sum_{j=0}^{l}\left[d_{j} \mid l_{\text {_backoffs }}\right]\left(1-p_{i}\right) p_{i}^{l} \\
= & \sum_{l=1}^{\infty}\left(\sum_{j=1}^{l} E\left[\left(k_{j}+1\right) \bar{F}+b_{j} \mid l_{-} \text {backoffs }\right]\right. \\
& \left.+E\left[\left(k_{1}+\frac{1}{2}\right) \bar{F}+b_{1} \mid l_{-} \text {backoffs }\right]\right)\left(1-p_{i}\right) p_{i}^{l} \\
& +E\left[\left(k_{1}+\frac{1}{2}\right) \bar{F}+b_{1} \mid l_{-} \text {backoffs }\right]\left(1-p_{i}\right) \\
= & \sum_{l=0}^{\infty} \sum_{j=0}^{l} E\left[(\lambda \bar{F}+1) b_{j}+\bar{F}\right]\left(1-p_{i}\right) p_{i}^{l} \\
& -\frac{\bar{F}}{2} \sum_{l=0}^{\infty}\left(1-p_{i}\right) p_{i}^{l}
\end{aligned}
$$

For the basic exponential backoff scheme, where $E\left[b_{j}\right]=$

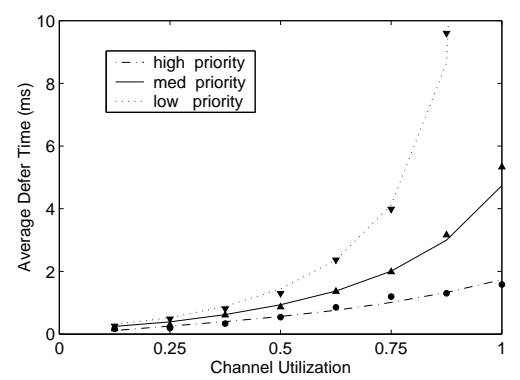

Figure 2: Delay Model Verifi cation (parameters are the same as in fi gure 1).

$\left(2^{j} \cdot C W_{\min }-1\right) / 2$, we have

$$
\begin{aligned}
d_{l}(\text { basic })= & \frac{\lambda_{i} F+1}{2} C W_{\min }\left[\frac{2^{m} p_{i}^{m+1}}{1-p_{i}}\right. \\
& \left.+\frac{1+p_{i}^{m}-\left(2^{m+1}+3\right) p_{i}^{m+1}-2 p_{i}^{m+2}}{1-2 p_{i}}\right] \\
& +\left[F-\frac{\lambda F+1}{2}\right] \frac{1}{1-p_{i}}-\frac{\bar{F}}{2}
\end{aligned}
$$

For our priority-based backoff scheme,

Hence, we have

$$
E\left[b_{j}\right]=\frac{\left(2^{j}+p_{i} \alpha_{i}\right) C W_{\min }-1}{2}
$$

$$
\begin{aligned}
& d_{l}=d_{l}(\text { basic })+\left[\sum_{l=m+1}^{\infty} \sum_{j=0}^{m} p_{i} \alpha_{i}+\sum_{l=0}^{m} \sum_{j=0}^{l} p_{i} \alpha_{i}\right] \\
& \times\left(\frac{\lambda_{i} \bar{F}+1}{2}\right)\left(1-p_{i}\right) p_{i}^{l} \\
& =d_{l}(\text { basic })+p_{i} \alpha_{i} \frac{\lambda_{i} \bar{F}+1}{2} \frac{p_{i}\left(1-p_{i}^{m}\right)}{1-p_{i}} \cdot C W_{\text {min }}
\end{aligned}
$$

Eq. (22) gives the average of the total accumulated deferred time for a packet transmission when backoff occurs. When the channel is sensed free and the fbw transmits without backoff, the deferred time is zero if transmission succeeds or $\left(\bar{F}+d_{l}\right)$ if a collision happens. Similar to Eq. (10),

$$
d_{\text {defer }, i}=P_{\text {free }, i}\left(1-e^{-\lambda_{i}}\right)\left(\bar{F}+d_{l}\right)+P_{\text {busy }, i} d_{l}
$$

Let $d_{\text {transmission, } i}$ denote the transmission time of a packet, whose average is $F_{i}$, then the average service delay is

$$
d_{\text {service }, i}=d_{d e f e r, i}+d_{\text {transmission }, i}
$$

Eq. (24) is the result for head-of-line packets. When queuing delay is considered, the total delay can be obtained by utilizing the delay results of an M/M/1 queue [4]. Specifi cally, suppose the traffi $\mathrm{c}$ arrival rate is Poisson distributed with average rate of $R_{\text {arrival }, i}$, and the service rate is also Poisson distributed with average rate of $R_{\text {service }, i}=1 / d_{\text {service }, i}$. Then the total delay is

$$
d_{i}=1 /\left(R_{\text {service }, i}-R_{\text {arrival }, i}\right)
$$

Eq. (25) can be used for admission control to check whether the delay bound of the fbw is satisfi ed.

Figure 2 shows the comparison between the analytical model and the simulation results for the average packet service latency (excluding queuing delay) as the traffi c load increases. The lines represent the numerical results calculated using the model, while the symbols indicate the simulation results. We can see that the simulation and analytical results are close to each other, thereby verifying our analysis. 


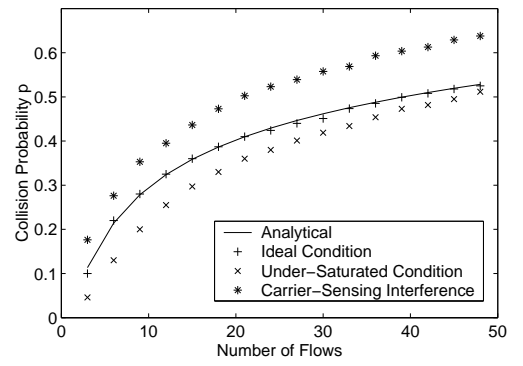

Figure 3: Impact of under-saturated nodes and hidden terminals.

\subsection{MBRP in Real-world Environments}

\subsubsection{Impact of Under-Saturated Nodes}

The basic MBRP mechanism described in section 3.1 assumes nodes are saturated, i.e., all nodes in the network have packets in their queue for transmission. When nodes are not saturated, our analysis may overestimate the actual number of collisions in the network. Figure 3 shows the impact of under-saturated nodes. The line represents the collision possibility calculated through the analysis in section 3.1.1 versus the number of competing fbws, assuming all nodes are saturated. The symbols that match the line are the simulation results with all nodes saturated. The lower symbols are the measurement results for simulations with a certain percentage $(30 \%)$ of unsaturated nodes, while the remaining nodes are operated in saturated conditions. The difference indicates that when nodes are not saturated, the basic MBRP analysis overestimates the collision rate in the network. This leads to lower throughput and higher calculated delay.

\subsubsection{Impact of Hidden Terminals}

Another assumption used by MBRP is ideal channel conditions, i.e., no packet corruption, and no hidden terminals. It does not consider the fact that a node's carrier-sensing neighbors can also interfere with its transmission, even though the node cannot correctly decode the interfering packets. The impact of interfering nodes is thus not reftected in the fbw set. Hence, the modeling analysis may underestimate the actual collisions in the network. This is especially true in a multihop network where the hidden terminal problem and carrier sensing interference become more signifi cant.

Consider a simple topology as shown in fi gure 4, where node 3's transmission interferes with node 2's packet reception because the nodes are with carrier-sensing range of each other. Flows $f_{1}$ and $f_{2}$ then confict. Because nodes 2 and 3 cannot decode each other's packets correctly, node 1 is unaware of the existence of node 3 . Consequently node 1 will not include fbw $f_{2}$ in its fbw set. ${ }^{2}$ The upper symbols in figure 3 show the impact of the interference from the nodes in the carrier sensing range but out of the transmission range of a node. With the interference from carrier-sensing neighbors, the basic MBRP analysis underestimates the collisions in the network.

\footnotetext{
${ }^{2}$ If nodes 2 and 3 are direct neighbors, node 2 will include fbw $f_{2}$ in its fbw set. Node 1 will thereby learn of the interference.
}

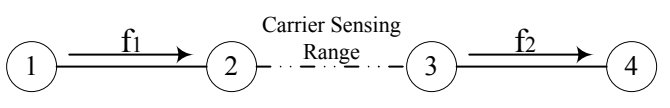

Figure 4: Scenario with "hidden terminals".

\subsubsection{Adjustment using Measurement Feedback}

In addition to the above described assumptions, unexpected collisions, such as those caused by control packet transmissions and random interference (e.g., microwave or other wireless transmissions), will also affect the results. As shown in fi gure 3 , the presence of unsaturated nodes makes our model overestimate the channel usage, while unexpected packet loss due to hidden terminal, collision or interference makes our model underestimate the channel usage. Depending on the real network topology and traffi c distribution, these issues will result in a discrepancy between the model-based output and the actual measurement results.

To mitigate these effects, we improve MBRP by utilizing the difference between the measured value and the model output as run-time feedback to improve the accuracy of our model. The improved analysis is called Enhanced MBRP (EMBRP).

Suppose at the time when the $\mathrm{n}$-th fbw is admitted, the channel collision ratio estimated by new EMBRP model is $p_{\text {cal }}(n)$. When the $(\mathrm{n}+1)$-th fbw is requested, the real channel collision ratio measurement is $p_{\text {measure }}(n)$. The difference between the model result and actual value is then calculated:

$$
\Delta p(n)=p_{\text {measure }}(n)-p_{\text {cal }}(n)
$$

Hence, the difference $\Delta p(n)$ during the $\mathrm{n}$-th fbw request can then be used by the EMBRP model as feedback to make a better decision for the $(n+1)$-th request.

First, the basic MBRP model predicts the collision ratio $p_{\text {cal }}(n+1)^{\prime}$ based on current fbw information using the process in section 3.1. Then EMBRP combines the output from MBRP and $\Delta p(n)$ to produce the fi nal estimation $p_{\text {cal }}(n+1)$ as the following:

$$
p_{\text {cal }}(n+1)=p_{\text {cal }}(n+1)^{\prime}+k_{1} \Delta p(n)+k_{2} \sum_{i=1}^{n} \Delta p(i)
$$

where $k_{1}$ is the proportionality coeffi cient that controls the response to changes of collision rate. A larger $k_{1}$ improves the response rate, but it may lead to systematic error or oscillation. $k_{2}$ is the integration coefficient that decreases the response rate but diminishes system error. The adjustment of the values for $k_{1}$ and $k_{2}$ is important for the accuracy of our prediction. Because we do not expect the network state to dramatically change, previous measurements are weighted more heavily, and $k_{1}$ is generally smaller than $k_{2}$. We will explain in detail the parameter selection in the experiments in section 5 . Section 5 discusses estimation of the parameters in more detail.

Based on this adjusted collision rate, an adjusted $\lambda$ can be calculated. Specifi cally, from Eq.(3), we have

$$
\lambda_{i}=-\log \left(1-p_{i}\right)
$$


Consequently, adjusted $L\left(a_{i}\right)$ can be calculated using Eq.(10). Adjusted throughput and delay prediction can further be obtained.

The collision rate that a node experiences is measured as the probability that a packet transmission by the node in question fails using a standalone measurement process at the MAC layer. Given a measurement interval, the process continuously measures the collision rate without being triggered by the analytical model. When the latter needs the measurement result to adjust the calculation, it obtains the results from the measurement unit.

The collision rate is calculated as following: we count the number of failed transmissions, i.e., number of packets that do not receive ACK packets, and divide it by the total number of data transmissions in a given measurement duration. Hence, we have

$$
\bar{p}=N_{\text {unacked }} / N_{\text {transmitted }}
$$

We further use an ARMA (Auto Regressive Moving Average) [14] fi lter to provide run-time estimation, considering the previous results, to smooth the measurement. $\beta$ is the smoothing factor.

$$
\bar{p}^{\prime}=\beta \bar{p}+\frac{(1-\beta)}{n} \sum_{i=0}^{n-1} \bar{p}_{i}
$$

Note that the collision rate measurement is passive and it does not incur extra communication overhead, i.e., no packet transmission is needed. Additionally, the modeling and measurement results are only calculated at the source and intermediate nodes along a fbw path, i.e., the destination is not involved. This is because the interference at the reception node is included in the unacknowledged packet measurement of the node's upstream neighbor.

\section{Integration with Routing Protocols}

Our resource prediction model can be integrated with ad hoc routing protocols as a module sitting in between the IP routing layer and the MAC layer, as shown in fi gure 5.

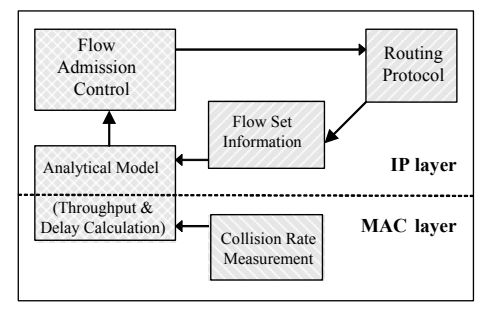

Figure 5: Integration with routing protocols at the IP and MAC layers.

MBRP provides channel statistics of a node's local contending area. However, because packet delivery often occurs in a multi-hop fashion, a local decision is not suffi cient for the setup of an entire transmission path. The interference among neighboring nodes makes the estimation of channel utilization more diffi cult. For example, in fi gure 6, the circles indicate the transmission range of each node. Node A's neighborhood includes B, B's neighbors include A and C, and both B and D are $\mathrm{C}$ 's neighbors. Suppose node A requests a new fbw using the path $A \rightarrow B \rightarrow C \rightarrow D$ to reach the destination. If the bandwidth consumption of the fbw is $x$, then the bandwidth consumption is actually $2 \times x$ for nodes $\mathrm{A}$ and $\mathrm{C}$, and $3 \times x$ at node $\mathrm{B}$. This is because nodes within transmission range of each other contend for the shared medium. Therefore, a new fbw will consume the resources in the neighborhood of all the nodes along the transmission path.

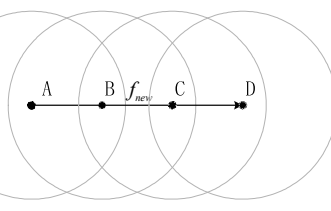

Figure 6: An example topology.

The routing process can be augmented to analyze the interference relationship among the nodes on the potential transmission path, as well as to disseminate the fbw information along the path. Then, based on the potential fbw set information, the estimated throughput or delay can be calculated using the analytical model described in the previous section. Finally, the source can choose the path that best meets the fbw's QoS requirement.

We brieffy describe how the model is integrated with reactive routing protocols, using AODV [19] as an example. The basic fbw setup process can be divided into a Request and a Reply phase. In the request phase, the source node sends RREQ messages for the new fbw, including QoS information such as the traffi c class of the fbw, the required quality, and the minimum throughput or accumulated delay through previous hops. Upon reception of the RREQ packet, each intermediate node adds a pending record for this fbw and rebroadcasts the RREQ if the fbw is locally admissible. This indicates that the predicted quality of the new fbw is within an acceptable range, i.e., the minimum available bandwidth along the path is larger than the fbw's throughput requirement, or the accumulated delay is smaller than the latency requirement. If the fbw is not locally admissible, the RREQ packet is dropped. After the propagation of RREQ packets, intermediate nodes use Neighbor Reply messages (NREP) to notify neighbors about the potential load, including the new fbw information. The updated fbw set information, disseminated by NREP packets, serves as the input of the analytical model as described in section 3.1.1. The RREQ packet reaches the destination if a path with satisfi ed quality exists.

If a RREQ message is received, the destination node sends a Route Reply message (RREP) along the reverse path to the source node during the reply phase. Intermediate nodes obtain updated neighbor load information through the NREP packets in the Request phase. They now recompute the quality of service that can be provided to the fbw and forward the RREP if the new fbw is locally admissible. The source node selects an optimal path based on the available levels of service. Once data packet transmission begins, the nodes along the propagation path also send NREP packets to notify their neighbors that the fbw has been admitted. Therefore, all nodes that are affected by the new fbw obtain updated channel utilization information. 
When path breaks due to node movement, route maintenance is performed so that the source node can re-discover a new valid route. In this case, fbw information is updated through the new setup process. In a highly mobile environment, frequent broken paths and neighbor nodes changes will result in stale fbw information and, consequently, inaccurate fbw quality estimation. However, it is likely that a valid and stable route rarely exists with the high mobility. We foresee that the integration of MBRP with routing protocols will be most effective in environments with low or controlled mobility. For instance, backhaul networks consisting of wireless routers that provide multi-hop communication for mobile nodes are well-suited for our model.

The above discussion describes how MBRP can be combined with reactive routing protocols. For proactive routing protocols, fbw set information can be exchanged between neighboring nodes through Hello messages or any other periodic neighbor link update messages. However, an extra call setup process is needed to accomplish the quality prediction. This is because the interference that will be caused by the new fbw cannot be determined by neighbor exchanges alone. The proposed MBRP mechanism can also be integrated with QoS routing protocols in a similar manner.

\section{Experimental Results}

We implemented our approach in the NS-2 [11] simulator with the Monarch mobility extensions [6]. A modifi ed MAC protocol is used to provide differentiated scheduling as described in [22]. $C W \min$ is set to 32 and $m$ is 5. The link bandwidth is set to $2 \mathrm{Mbps}$. The value of $\beta$ in the collision measurement (Eq.(30)) is set to 0.8 to place greater weight on recent measurements. The AODV routing protocol, modifi ed as described in section 4 , is utilized for multi-hop communication in the second and third sets of simulations.

\subsection{Single Broadcast Region}

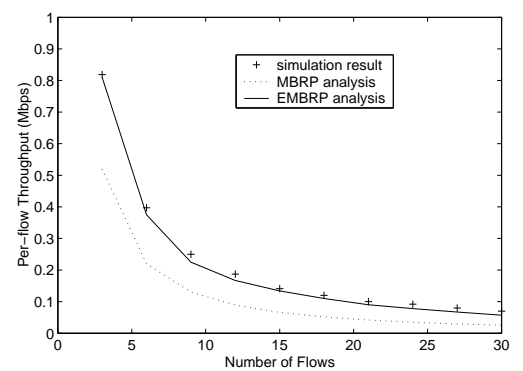

Figure 7: Throughput prediction with under-saturated nodes.

The fi rst set of simulations explores MBRP performance in a single-hop scenario, where a group of source and destination pairs are all within the same broadcast region. No interference from the carrier-sensing range neighbors or hidden terminals occurs in this scenario. All the fbws have the same priority where the priority adjustment parameter $\alpha$ equals 0 . The packet size is 1000 bytes. 1/3 of all fbws are operated at non-saturated condition, with a packet sending rate of 10 $\mathrm{pkt} / \mathrm{s}$, while the remaining fbws all have sending rate of 100 $\mathrm{pkt} / \mathrm{s}$. MBRP is not used for admission control in this set of experiments so that results with a large number of fbws can be shown.

Figure 7 shows the results of bandwidth prediction for each fbw. The symbol represents the simulation results for the average per-fbw throughput. The dotted line is the numerical result calculated using the basic MBRP model. The model results are lower than the actual throughput because of the overestimation of collision possibility as shown in fi gure 3. By using the measured collision rate to adjust the model calculation, EMBRP obtains results close to the simulation values.

The parameter $k_{1}$ in Eq.(27) is set to 0.2 , and $k_{2}$ is set to 0.8 . As explained in section $3.2, k_{2}>k_{1}$ is because more weight is given to previous results to achieve stability. As the results become more stable, the impact of $\Delta(p)$ approaches zero, while the impact of $\sum \Delta(p)$ becomes smaller because $k_{2}<1$. As more fbws are admitted, nodes experience longer service delay, and consequently longer queuing latency. Hence, the impact of the understaurated condition is reduced.

\subsection{Grid Topology}

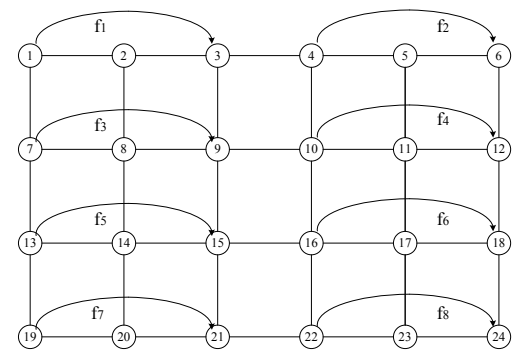

Figure 8: Grid topology.

This set of simulations examines the effectiveness of applying MBRP to admission control in a multi-hop network. Specifi cally, the nodes form a $4 \times 6$ grid with inter-node spacing of $200 \mathrm{~m}$, as shown in fi gure 8 . The parameters of the fbws are indicated in table 1 . For high priority VoIP fbws, we require delivery delay less than $100 \mathrm{~ms}$ as indicated by delay req. The minimum bandwidth requirement for high and low priority traffi c is $64 \mathrm{kbps}$ and $100 \mathrm{kbps}$, respectively, as indicated by $b w_{r e q}$. This traffic pattern represents a network environment where background traffi $\mathrm{c}$ is delay-tolerant while higher priority is given to traffi $\mathrm{c}$ with stringent real time constraints. In this set of experiments, we use the following admission policy: admit the maximum number of fbws while ensuring that their received quality of the service meets their service needs. All the fbws in fi gure 8 are started sequentially at 10 second intervals. Among them, fbws 2, 4, 6 and 8 are high priority, while the rest are low priority.

Table 1: Priority traffi c parameters.

\begin{tabular}{||c||c|c|c|c||}
\hline $\begin{array}{c}\text { Priority } \\
\text { Class }\end{array}$ & $\begin{array}{c}\text { Packet Size } \\
\text { (bytes) }\end{array}$ & $\begin{array}{c}\text { Rate } \\
\text { (Kbps) }\end{array}$ & $\begin{array}{c}\text { bweq } \\
\text { (Kbps) }\end{array}$ & $\begin{array}{c}\text { delay } y_{r e q} \\
(\mathrm{~ms})\end{array}$ \\
\hline High (G.711 VoIP) & 160 & 64 & 64 & 100 \\
\hline Low (CBR) & 500 & 200 & 100 & - \\
\hline
\end{tabular}

When there is no admission control, all fbws start at their scheduled time. When MBRP is applied, i.e., no measurement feedback is utilized, the first 7 fbws are all admitted, while the 8th fbw is rejected. When EMBRP is utilized, the 
Table 2: Throughput for fbws without admission control (Kbps).

\begin{tabular}{||c|c|c|c|c|c|c|c|c|}
\hline Start time & $f_{\mathbf{1}}$ & $f_{2}$ & $f_{3}$ & $f_{4}$ & $f_{5}$ & $f_{6}$ & $f_{7}$ & $f_{8}$ \\
\hline$t_{0}+50$ & 200 & 64 & 132 & $\underline{\mathbf{5 7}}$ & 198 & 64 & - & - \\
\hline$t_{0}+60$ & 200 & 64 & 112 & $\underline{\mathbf{5 6}}$ & 197 & 64 & 200 & - \\
\hline$t_{0}+70$ & 190 & 64 & $\underline{\mathbf{9 8}}$ & $\underline{\mathbf{5 1}}$ & 114 & $\underline{\mathbf{5 0}}$ & 198 & 64 \\
\hline
\end{tabular}

Table 3: Throughput for MBRP admitted fbws (Kbps).

\begin{tabular}{||c|c|c|c|c|c|c|c|c|}
\hline Start time & $f_{\mathbf{1}}$ & $f_{2}$ & $f_{3}$ & $f_{4}$ & $f_{5}$ & $f_{6}$ & $f_{7}$ & $f_{8}$ \\
\hline$t_{0}+50$ & 200 & 64 & 132 & $\underline{\mathbf{5 7}}$ & 200 & 64 & - & - \\
\hline$t_{0}+60$ & 194 & 64 & 112 & $\underline{\mathbf{5 5 . 7}}$ & 194 & 64 & 197 & - \\
\hline$t_{0}+70$ & 194 & 64 & 112 & $\underline{\mathbf{5 5 . 7}}$ & 193 & 64 & 197 & - \\
\hline
\end{tabular}

6th fbw is rejected, while the rest are all admitted. Tables 24 show the throughput of the admitted fbws with each of the different schemes. The underlined values indicate that the fbw does not receive its required quality. When there is no admission control (as shown in table 2), the throughput after time $t_{0}+50$ decreases signifi cantly for fbws that experience more contention, i.e., $f_{3}$ through $f_{6}$. When admission control is utilized, seven fbws are admitted; however, the fbws receive different quality, as indicated in tables 3 and 4 . Flows admitted by EMBRP in general have higher throughput than that of MBRP. In particular, when $f_{6}$ is admitted using MBRP, the throughput of $f_{4}$ falls below $64 \mathrm{kbps}$, resulting in poor quality. When the measured collision rate is fed back into the model calculation, EMBRP achieves better performance because the impact of hidden terminal and carrier-sensing neighbors are included in the calculation.

Figure 9 shows the average packet latency of the high priority fbws after all fbws started. When there is no admission control, both $f_{4}$ and $f_{6}$ fail to meet their delay requirement. When admission control with MBRP is used, $f_{4}$ does not receive required quality. When EMBRP is utilized, all admitted fbws have average latency below $100 \mathrm{~ms}$. Figure 10 further illustrates the packet delivery latency for the admitted high priority fbws using EMBRP. All the admitted fbws have average latency of less than $100 \mathrm{~ms}$. The occasional surge of latency for the fbws is caused by the temporary fboding of routing control packets. Because we place more weight on the previous collision rate than the current rate, this occurrence is short-lived. Specifi cally, the feedback parameter $k_{1}$ is set to 0.2 as in the first set of experiments, and $k_{2}$ is set to 1.1 . The intuition of choosing this value is that as more fbws are admitted in the network, more interference from carrier-sensing neighbors and hidden terminals occur. The difference between the measurement and analytical results therefore increases. We also examine the value of $k_{2}$ with different topology and traf-

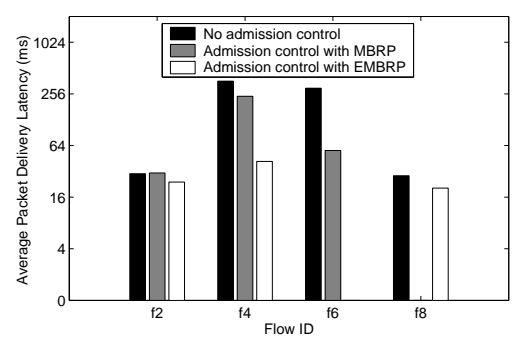

Figure 9: Average packet delivery latency.
Table 4: Throughput for EMBRP admitted fbws (Kbps).

\begin{tabular}{||c|c|c|c|c|c|c|c|c|}
\hline Start time & $f_{\mathbf{1}}$ & $f_{2}$ & $f_{3}$ & $f_{4}$ & $f_{5}$ & $f_{6}$ & $f_{7}$ & $f_{8}$ \\
\hline$t_{0}+50$ & 198 & 64 & 186 & 64 & 198 & - & - & - \\
\hline$t_{0}+60$ & 194 & 64 & 174 & 64 & 196 & - & 200 & - \\
\hline$t_{0}+70$ & 190 & 64 & 166 & 64 & 186 & - & 200 & 64 \\
\hline
\end{tabular}

fi c scenarios, for positive $\Delta p, k_{2}=1.1$ in general can achieve fairly accurate and stable prediction.

\subsection{Random Topology}

To better understand the performance of MBRP, we now examine random topologies. In this set of simulations, we generate a random topology in a $1000 m \times 1000 m$ area with 50 nodes. Flows are randomly chosen between node pairs and the traffi c parameters are the same as described in table 1. Ten low priority fbws are started at the beginning of the simulations and are used as background traffi c. We then increase the number of high priority fbws at 10 second intervals. The average path length is 2.7 . Because the impact of hidden terminals and carrier-sensing interference is more signifi cant than in the under-saturated conditions in a multi-hop environment, $k_{1}$ and $k_{2}$ are set to 0.2 and 1.1 , respectively.

Table 5: Admission results with high priority fbws.

\begin{tabular}{||c|c|c|c|c|c|c|c|c|c|c||}
\hline Flow ID & 1 & 2 & 3 & 4 & 5 & 6 & 7 & 8 & 9 & 10 \\
\hline accept & $\checkmark$ & $\checkmark$ & $\checkmark$ & $\checkmark$ & $\checkmark$ & $\checkmark$ & $\checkmark$ & $\times$ & $\checkmark$ & $\times$ \\
\hline
\end{tabular}

Table 5 shows the fbw admission results using EMBRP. The 8th high priority fbw is rejected because its delay requirement cannot be satisfied. The 10th fbw is rejected because, if it was admitted, the quality of service of the other fbws would degrade unacceptably.

Figure 11 shows the average packet delivery latency (in logarithmic scale) for the high priority fbws with and without admission control. The data points represent the average delay of all the fbws, while the error bars indicate the maximum and minimum delay among the fbws. For instance, at $80 \mathrm{sec}-$ onds, the average delay of the 7 admitted fbws ( $f_{8}$ is rejected) is $41.2 \mathrm{~ms}$, while the maximum delay of one fbw $\left(f_{4}\right)$ is 55.2 $\mathrm{ms}$, and the lowest of a fbw $\left(f_{5}\right)$ is $30 \mathrm{~ms}$. When no admission control is performed, at time 80 s the average delay of the $8 \mathrm{fbws}$ is $82.7 \mathrm{~ms}$. However, the service needs of one of the fbws $\left(f_{8}\right)$ cannot be met; its delay is $127.7 \mathrm{~ms}$. Similar events occur at time 100 s, where $f_{10}$ is rejected when EMBRP is used. This indicates that by predicting the per-fbw quality, EMBRP assists the fbw admission decision so as to meet the needed service quality constraints. Note that we shifted the

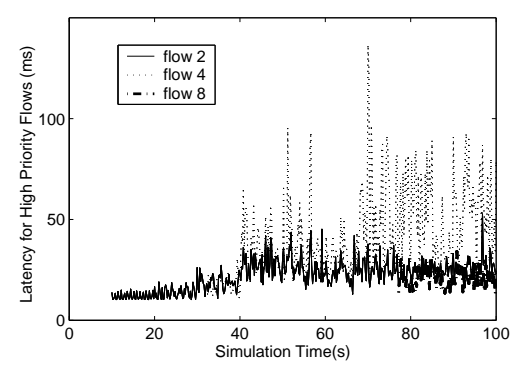

Figure 10: Packet delivery latency for admitted high priority fbws. 


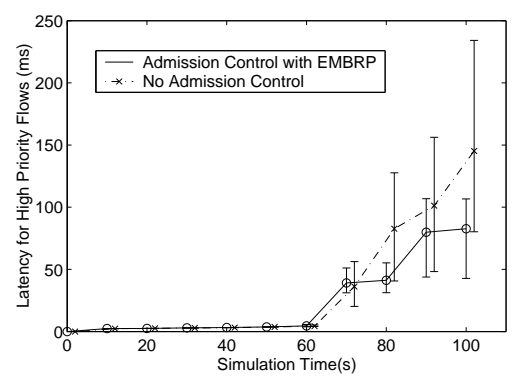

Figure 11: Average Latency for all admitted fbws.

dotted lines for the results without admission control so that the error bars do not overlap.

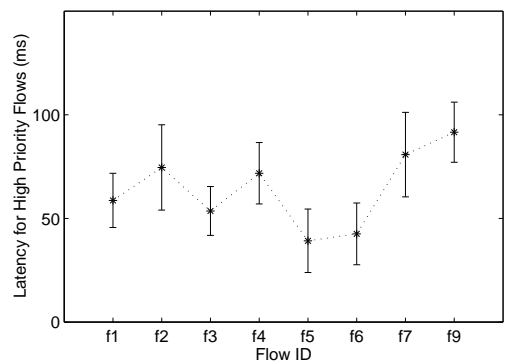

Figure 12: Packet delivery latency for admitted high priority fbws.

Figure 12 further shows the packet delivery latency for each admitted high priority fbw when admission control is applied. The data points are the average delay of each fbw after time 100 s; no new fbw is admitted after that time. The error bars indicate the $95 \%$ confi dence interval of the delay for each individual fbw. The results show that the quality of service needs of each fbw is met, thereby verifying the effectiveness of EM$\mathrm{BRP}$ as an admission control solution.

\section{Conclusion}

This paper proposes a model-based resource prediction mechanism that supports real time communication in multi-hop wireless networks. An analytical model for differentiated MAC scheduling protocol is given, with adjustments for the multi-hop environment. The model can predict per-fbw and system-wide throughput and delivery latency, thereby enabling admission control of the fbws and providing an effi cient network management utility. This is benefi cial in the deployment of a real ad hoc network where knowledge of resource allocation and consumption is needed to meet the service requirements.

\section{References}

[1] I. Ada and C. Castelluccia. Differentiation Mechanisms for IEEE 802.11. In Proceedings of the IEEE Conference on Computer Communications (INFOCOM), Anchorage, Alaska, April 2001.

[2] G.-S. Ahn, A. Campbell, A. Veres, and L.-H. Sun. Supporting Service Differentiation for Real-Time and Best-Effort Traffi c in Stateless Wireless Ad Hoc Networks (SWAN). IEEE Transactions on Mobile Computing, 1(3):192-207, July-Septemeber 2002.
[3] M. Barry, A. T. Campbell, and A. Veres. Distributed Control Algorithms for Service Differentiation in Wireless Packet Networks. In Proceedings of the IEEE Conference on Computer Communications (INFOCOM), Anchorage, Alaska, 2001.

[4] O. Bertsekas and R. Gallager. Data Networks, 2nd Edition. Prentice -Hall, 1992

[5] G. Bianchi. Performance Analysis of the IEEE 802.11 Distributed Coordination Function. IEEE Journal on Selected Areas in Communications, 18(3):535-547, March 2000.

[6] J. Broch, D. A. Maltz, D. B. Johnson, Y.-C. Hu, and J. Jetcheva. A Performance Comparison of Multihop Wireless Ad Hoc Network Routing Protocols. In Proceedings of the $4^{\text {th }}$ ACM/IEEE International Conference on Mobile Computing and Networking (MobiCOM'98), pages 85-97, Dallas, TX, October 1998.

[7] R. Carter and M. Crovella. Measuring Bottleneck Link Speed in Packetswitched Network. Technical Report BU-CS-96-006, Computer Science Department, Boston University, March 1996.

[8] I. Chakeres and E. Belding-Royer. PAC: Perceptive Admission Control for Mobile Wireless Networks. Submitted for publication.

[9] S. Chen and K. Nahrstedt. Distributed Quality-of-Service Routing in Ad-Hoc Networks. IEEE Journal of Selected Areas in Communications, 17(8), August 1999.

[10] D.Grossman. New Terminology and Clarifi cations for Diffserv. Request For Comments (Draft Stardard) 3260, Internet Engineering Task Force, April 2002

[11] K. Fall and K. Varadhan. ns Manual. http://www.isi.edu/nsnam/ns/doc/. The VINT Project.

[12] J. Goodman and A. Greenberg. Stability of Binary Exponential Backoff. Journal of the ACM, 35(3), March 1998.

[13] IEEE. 802.11e Draft 3.1, May 2002.

[14] S. M. Kay. Modern Spectral Estimation. Prentice -Hall, 1988.

[15] H. Kim and J. C. Hou. Improving Protocol Capacity with Model-based Frame Scheduling in IEEE 802.11-operated WLANs. In Proceedings of the Ninth Annual International Conference on Mobile Computing and Networking (MobiCOM'03), pages 190-204, San Diego, CA, September 2003.

[16] D. Maltz. Resource management in multi-hop ad hoc networks. Technical Report CMU CS 00-150, School of Computer Science, Carnegie Mellon University, July 2000.

[17] K. Nichols and B. Carpenter. Defi nition of Differentiated Services Per Domain Behaviors and Rules for Their Specifi cation. Request For Comments (Draft Stardard) 3086, Internet Engineering Task Force, April 2001.

[18] V. Paxson. End-to-End Internet Packet Dynamics. In Proceedings of the ACM SIGCOMM Conference on Communications Architectures, Protocols and Applications, pages 138-152, Cannes, France, September 1997.

[19] C. E. Perkins and E. M. Royer. Ad-hoc On-Demand Distance Vector Routing. In Proceedings of the $2^{\text {nd }}$ IEEE Workshop on Mobile Computing Systems and Applications, pages 90-100, New Orleans, LA, February 1999.

[20] R. S. Prasad, M. Murray, C. Dovrolis, and K. Claffy. Bandwidth Estimation: Metrics, Measurement Techniques and Tools. IEEE Network, 17(6), November 2003.

[21] S. Shenker, C.Partridge, and R. Guerin. Specifi cation of Guaranteed Quality of Service. Request For Comments (Draft Stardard) 2212, Internet Engineering Task Force, September 1997.

[22] Y. Sun, E. Belding-Royer, X. Gao, and J. Kempf. A Priority-based Distributed Call Amission Protocol for Multi-hop Wireless Ad hoc Networks. Technical report, Computer Science Department, UC Santa Barbara, April 2004.

[23] A. Veres, A. T. Campbell, M. Barry, and L.-H. Sun. Supporting Service Differentiation in Wireless Packet Networks Using Distributed Control. IEEE Journal of Selected Areas in Communications, 19(10), October 2001.

[24] Y. Yang and R. Kravets. Contention-aware Admission Control for Ad hoc Networks. Technical Report 2003-2337, Computer Science Department, University of Illinois at Urbana-Champaign, April 2003. 